\title{
MONITORING SEABIRDS AND MARINE MAMMALS BY GEOREFERENCED AERIAL PHOTOGRAPHY
}

\author{
G. Kemper ${ }^{\text {a }}$ *, A. Weidauer ${ }^{\text {b }}$, T. Coppack ${ }^{\text {b, c }}$ \\ a GGS - Büro für Geotechnik, Geoinformatik und Service, Speyer, Germany - kemper@ggs-speyer.de \\ ${ }^{b}$ Institute of Applied Ecology (IfAÖ GmbH), Rostock, Germany - weidauer@ifaoe.de \\ ${ }^{\mathrm{c}}$ Current affiliation: APEM Ltd, Manchester, United Kingdom - t.coppack@apemltd.co.uk
}

KEY WORDS: Biological Monitoring, Digital Surveys, Environmental Impact Assessment, Marine Wildlife, Offshore Wind

\begin{abstract}
:
The assessment of anthropogenic impacts on the marine environment is challenged by the accessibility, accuracy and validity of biogeographical information. Offshore wind farm projects require large-scale ecological surveys before, during and after construction, in order to assess potential effects on the distribution and abundance of protected species. The robustness of site-specific population estimates depends largely on the extent and design of spatial coverage and the accuracy of the applied census technique. Standard environmental assessment studies in Germany have so far included aerial visual surveys to evaluate potential impacts of offshore wind farms on seabirds and marine mammals. However, low flight altitudes, necessary for the visual classification of species, disturb sensitive bird species and also hold significant safety risks for the observers. Thus, aerial surveys based on high-resolution digital imagery, which can be carried out at higher (safer) flight altitudes (beyond the rotor-swept zone of the wind turbines) have become a mandatory requirement, technically solving the problem of distant-related observation bias. A purpose-assembled imagery system including medium-format cameras in conjunction with a dedicated geo-positioning platform delivers series of orthogonal digital images that meet the current technical requirements of authorities for surveying marine wildlife at a comparatively low cost. At a flight altitude of $425 \mathrm{~m}$, a focal length of $110 \mathrm{~mm}$, implemented forward motion compensation (FMC) and exposure times ranging between $1 / 1600$ and $1 / 1000 \mathrm{~s}$, the twin-camera system generates high quality 16 bit RGB images with a ground sampling distance (GSD) of $2 \mathrm{~cm}$ and an image footprint of $155 \times 410 \mathrm{~m}$. The image files are readily transferrable to a GIS environment for further editing, taking overlapping image areas and areas affected by glare into account. The imagery can be routinely screened by the human eye guided by purpose-programmed software to distinguish biological from non-biological signals. Each detected seabird or marine mammal signal is identified to species level or assigned to a species group and automatically saved into a geo-database for subsequent quality assurance, geo-statistical analyses and data export to third-party users. The relative size of a detected object can be accurately measured which provides key information for species-identification. During the development and testing of this system until 2015, more than 40 surveys have produced around 500.000 digital aerial images, of which some were taken in specially protected areas (SPA) of the Baltic Sea and thus include a wide range of relevant species. Here, we present the technical principles of this comparatively new survey approach and discuss the key methodological challenges related to optimizing survey design and workflow in view of the pending regulatory requirements for effective environmental impact assessments.
\end{abstract}

\section{INTRODUCTION}

\subsection{General Introduction}

The marine environment is subject to a variety of anthropogenic influences, including global climate change. The environmental changes caused by climate change and the actions taken by humans to mitigate and to adapt to these changes are currently leading to an increased exploitation of marine resources with presumed (although largely unquantified) effects on marine life. The development of the offshore wind industry, for example, in combination with shipping and fisheries potentially leads to a reduction undisturbed wintering and resting areas for seabirds (Mendel \& Garthe, 2010; Dierschke et al., 2012). Furthermore, the increasing demand for marine gravels and sands intended for coastal protection is in potential conflict with the habitat requirements of some benthivorous duck species, which feed from molluscs and other invertebrates on the seabed (Müncheberg et al., 2012).
In order to determine the state of the marine environment in a rapidly changing world, as well as to assess the conservation status of its inhabitants, an increasingly accurate information base on the distribution and abundance of marine species is required. This entails effective monitoring schemes that provide meaningful data and detailed vulnerability assessment maps to inform policy decisions and to guide spatial planning procedures.

An objective evaluation of the environmental consequences of human activities for marine organisms has often been hampered by the lack of precise data in the affected areas. Here, we review recent developments to improve data acquisition by using highresolution georeferenced digital photography to map seabirds and marine mammals.

\subsection{Limitations of visual survey methods}

The assessment of the effects of offshore wind farms, for example, on seabirds and marine mammals are typically based

* Corresponding author 
on large-scale surveys that take place on a monthly or seasonal basis before, during and after construction of the wind farm. In the past, birds and mammals were usually counted by observers from ships (Garthe et al., 2002) or low-flying aircraft (Diederichs et al., 2002; Thomsen et al., 2004) along linear transects, thereby recording the frequency of observed objects and estimating the distance of each object from the point of observation. While ship-based counts are associated with considerable costs for logistics, airborne observations are comparatively time- and cost-effective, however pose methodological problems. Due to the relatively high airspeed of the deployed aircraft (about $100 \mathrm{kn} ; \sim 50 \mathrm{~m} / \mathrm{s}$ ) flying at around $250 \mathrm{ft}(\sim 80 \mathrm{~m})$ to ensure that bird species can be identified by the human eye, observer-based aerial surveys can provide only rough population estimates, especially in bird species that aggregate in large numbers. This is the case, for example, in the Baltic Sea where large groups of sea ducks occur. Low-flying aircraft also scare-off sensitive bird species to a non-negligible extent, such that quantitative estimates are additionally biased. Furthermore, the detection probability decreases with the distance between the bird and the observer. The resulting quantitative estimates and the quality of species identification can also vary widely between individual observers. Overall, these sources of variance that impact quantitative population estimates can only be compensated by synthetic correction factors. However, such corrections do not enhance the quality of the raw data per se and limit the comparability of data sets collected under different sampling configurations and conditions.

Assessing the environmental impacts of an offshore wind farm on birds essentially requires an unbiased detection of the distribution of individuals relative to the turbines' coordinates. If sensitive bird species are systematically disturbed by the detection method itself (low-flying aircraft, ship) cause-effect relationships between wind turbines and birds cannot be reliably addressed. Raw data resulting from observer-based surveys cannot be quality-assured retrospectively and used to secure evidence. Finally, there is a great safety risk for the human observer flying at low altitude past operational wind turbines.

\subsection{Aerial digital survey methods}

For the reasons mentioned above, survey methods based on aerial digital imaging by means of aircraft flying at significantly higher altitude are an effective alternative and are more and more replacing traditional observer-based survey techniques. The current version of the standard investigation concept (StUK4) of the German Federal Maritime and Hydrographic Agency (BSH) in fact recommends the use of aerial digital imaging methods for assessing the impact of offshore wind turbines on seabirds and marine mammals which has stimulated further refinement of this method.

In the early 1950s, the first attempts were made to quantify large aggregations of birds by conventional aerial photography. It was none other than the famous zoologist Bernhard Grzimek who demonstrated together with his son that large flocks of flamingos in Africa could only be quantified exactly on aerial photographs (Grzimek \& Grzimek, 1960). For the large-scale quantification of pelagic marine species, however, it was for a long time not feasible to use (analogue) aerial photography because of the sheer number of required images and the costs involved in image archiving. With the advent of digital camera technology and the availability of large digital archives, these problems are now manageable and the costs have become moderate. In addition, digital images provide the possibility of automated data analysis via image processing applications. Meanwhile, the development of digital aerial photography and the corresponding storage and computing capacities are so advanced that digital aerial imaging can be used standardized and cost-effectively.

Digital survey techniques have been increasingly used since about 2007 in environmental impact studies for the offshore wind industry, most notably in Denmark and the United Kingdom (Thaxter \& Burton, 2009). Both videographic and photographic techniques are deployed. Videography benefits from a higher frame rate though at the cost of a smaller frame (foot-print) size per camera unit. As a consequence, multiple parallel video streams are recorded to achieve sufficient areal coverage. High-resolution digital photography, on the other hand, compromises frame rate in favour of a larger aspect ratio per camera unit. Currently, the use of high-resolution mediumformat cameras with up to 80 megapixels is possible (Coppack et al. 2015). This enables photographic flights at altitudes over $1300 \mathrm{ft}(\sim 400 \mathrm{~m})$ with a ground sampling distance (GSD) of 2 $\mathrm{cm}$, depending on the specifications of the lens. At these altitudes, which are well above the rotor-swept zone of the wind turbines, displacement effects on sensitive bird species through the presence of the aircraft are significantly reduced. Furthermore, bird distributions and individual distances to manmade structures (wind turbines, vessels) can be accurately measured and stored digitally for further GIS analyses. This is a major advantage over observer-based protocols that involve voice recordings of quantitative estimates.

\section{DEVELOPMENT OF A METHOD BASED ON GEOREFERENCED PHOTOGRAPHY}

\subsection{Methodological principles}

Comparisons of simultaneous observer-based aerial surveys with digital aerial surveys generally show that relevant bird and marine mammal species can be detected and classified to species level in digital images (Thaxter \& Burton, 2009; Kulemeyer et al. 2011; Taylor et al. 2014, Dittmann et al. 2014). Although digital still images of birds and marine mammals initially showed significant motion blur due to long exposure times relative to the required airspeed and the lack of forwards motion compensation (FMC), an evaluation of the quantitative outcomes of conventional and digital surveys was possible. Results from a pilot study carried out by Kulemeyer et al. (2011) suggested that the numbers of three sea duck species had been significantly underestimated by the observer-based method, i.e., frequencies of individuals were 15\% (Common Eider), 69\% (Long-tailed Duck), and 98\% (Common Scoter) lower than frequencies determined by the aerial digital method (Kulemeyer et al., 2011). Such differences may be partly explained by the time lag between surveys and differences in coverage between photographic and visual methods. However, comparative results strongly suggest that photographic methods provide more accurate population estimates than conventional observer-based aerial surveys that are more susceptible to species-specific variation in flush behaviour and observation bias. To calibrate both methods accurately, it would be necessary to repeat such parallel flights under a range of different weather conditions.

\subsection{Camera technology}

Airborne image acquisition for biological monitoring is presently based on commercially available, photogrammetric components. The requirements to be met by a purpose- 
assembled camera system include a flight altitude of at least $1300 \mathrm{ft}$ (taking human flight safety, average cloud base and the escape distance of birds into account), a GSD of $2-3 \mathrm{~cm}$, a covered strip width of about $400 \mathrm{~m}$, and the option for a $30 \%$ to $40 \%$ image overlap to compensate for loss of effective coverage due to glint and glare (reflections from the sea surface), and vignetting (Figure 1; cf. Groom et al., 2013).

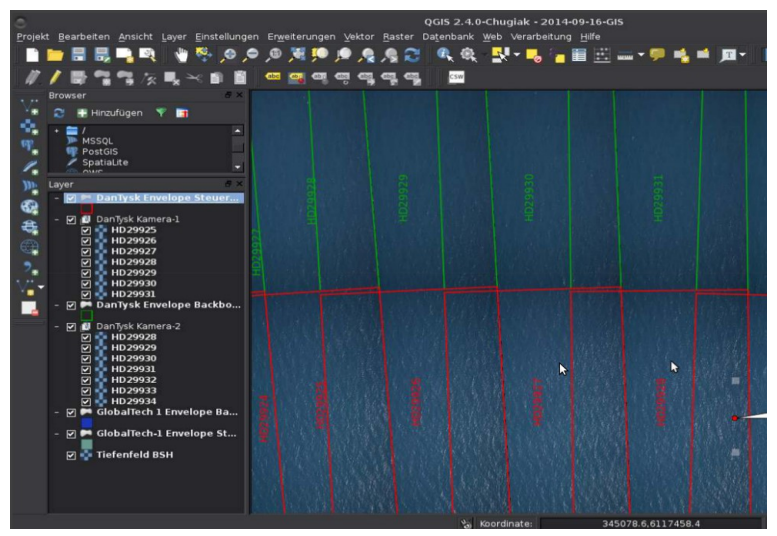

Figure 1. Example of a series of georeferenced, overlapping aerial images shown in QGIS

A typical camera system to monitor marine wildlife may consist of two or more medium format cameras (e.g. PhaseOne IXA180) mounted onto a gyroscopically stabilized platform (Figure 2).
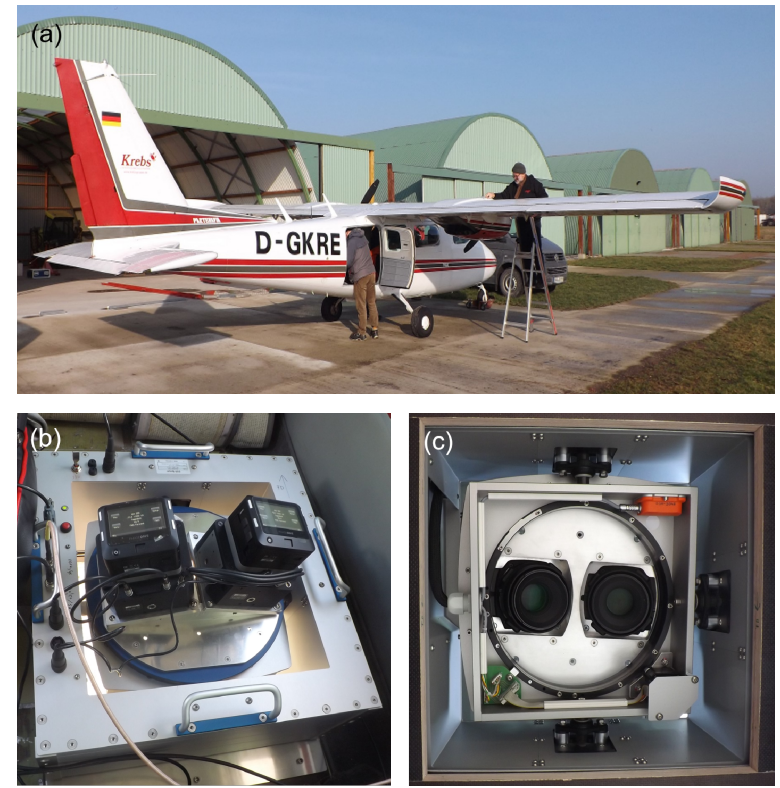

Figure 2. (a) Equipping a twin-engine aircraft for aerial digital surveys of marine wildlife, (b) View of a crosswise mounted tandem camera (PhaseOne IXA180) within a gyroscopically stabilized platform (GGS Speyer), (c) Exterior view of the camera system through the hatch of the aircraft

The gyro-static camera platform is connected with a computer system that triggers shutter release, stores the incoming image files and, at the same time, operates the flight management system (AeroTopoL) and the logging of geographic position and altitude from the GNSS-INS sensors (Kemper, 2012).

The sensors of each camera unit (PhaseOne IXA180) includes $10,320 \times 7,752$ cells at a resolution of 5.2 micron. Equipped with $110 \mathrm{~mm}$ lenses, this camera setup covers a strip width of $407 \mathrm{~m}$ from an altitude of $1400 \mathrm{ft}(423 \mathrm{~m})$ with $2 \mathrm{~cm} \mathrm{GSD}$. The minimal reading interval is $1.5 \mathrm{~s}$ and enables a theoretical image overlap of $48 \%$ at an airspeed of $100 \mathrm{kn}(\sim 50 \mathrm{~m} / \mathrm{s})$ and an image length in the direction of flight of $155 \mathrm{~m}$. Realistically, frame rates of $1.8-2.0 \mathrm{~s}$ are feasible and an image overlap of $30 \%$ sufficient under normal weather conditions. In addition, the camera system has implemented FMC. Trials have shown that details of birds and marine mammals are depicted best with sensitivity set to ISO 100, an aperture of 3.2 and an exposure of $<1 / 1,000 \mathrm{~s}$. In general, modular imaging systems based on medium format camera units are smaller and lighter and can be installed in small aircraft, including unmanned aerial vehicles (UAV). A current disadvantage of the applied camera unit is the unavailability of a near infra-red (NIR) band, which could be advantageous to compensate glare at sensor level. This option currently has to be solved by including a third dedicated NIR camera.

\subsection{Data storage and processing}

The monitoring guideline of the German Federal Maritime and Hydrographic Agency (BSH) currently recommend study areas of at least $2000 \mathrm{~km}^{2}$ of which normally $10 \%$ are to be sampled within one day. The volume of data (raw image files) arising from such surveys may reach around 5,000 $-8,000$ frames per flight and camera. Each camera can store about 500 - 800 GB on two separate solid-state disks during the flight. The image

transfer rate lies at $40-70 \mathrm{MB} / \mathrm{s}$ via USB 3.0 interface. Each raw image is unpacked and converted into orthorectified block oriented 500 - 800 MB large 16-bit RGB TIFF. The image files are augmented with acquisition-related metadata. After this georeferencing, the overlapping image areas are marked and edited, and series of analysable images are compiled. From this point on, visual screening of the image files with commercially available or open-source geographic information systems is possible. The sheer extent of the image database (a single flight takes up to $9 \mathrm{~TB}$ ) and the need to incorporate image processing algorithms into the workflow, puts forth the development and use of purpose-programmed software applications.

\subsection{Image analysis for object recognition}

There are basically two (not mutually exclusive) approaches to analysing and evaluating the resultant extensive image material: (1) automated image pre-processing (e.g. by eCognition; cf. Groom et al., 2013), including information from a visually screened subsample of images; (2) visual pre-processing of the complete image material with the help of purpose-programmed GIS-based screening applications (e.g. by combining QGIS and OpenCV; cf. Coppack et al. 2015, Figure 3).

Both approaches involve image pre-processing, in which the images without biological positive signals are sorted out and the geo-positions of all potential biological signals are collected in a database for subsequent identification and quality assurance.

The visual pre-selection of entire sets of images is presently the more robust approach, yet requires a lot of manpower. Visual screening is facilitated by purpose-programmed software applications, by which each image is divided into equal segments (50 - 80 segments per image, depending on effective footprint size) that can be tabbed through in a logical sequence 
(Figure 3). All objects that could potentially be a bird or a marine mammal (and any other conspicuous object) are marked manually on the screen and automatically stored in a database, thereby saving an image identification number, the flight transect number, the camera identification number, the object class and the geo-position of the object. The object class is roughly defined as: (1) swimming bird; (2) flying bird; (3) marine mammal; (4) conspicuous unknown object; (5) glare/glint; (6) wave/spray. For quality assurance, a sub-set of the images is screened twice. All selected objects from the visual screening process are then classified by an experienced analyst to reject objects that are not birds or marine mammals.

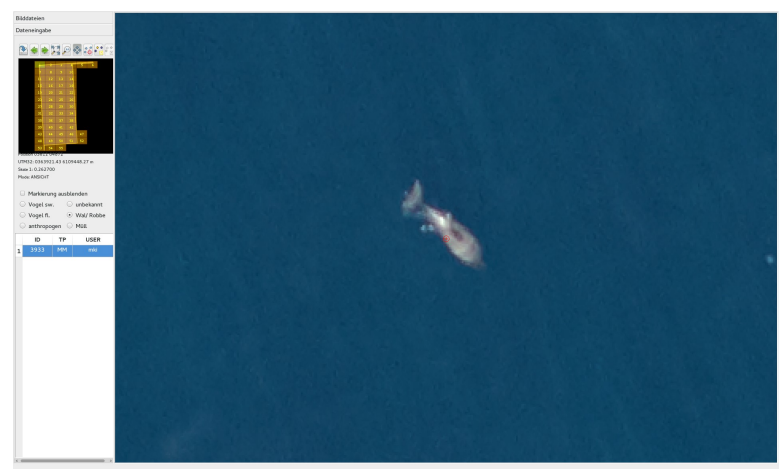

Figure 3. Screenshot of a software interface for the systematic visual screening of geo-referenced aerial photographs (example image shows a harbour porpoise)

\subsection{Species identification (ID) and quality assurance}

This subsequent step includes the identification of objects down to species or species-group level and the quantification of identified birds or marine mammals. Ideally, two experts classify each object independently, using a specially developed identification tool which is linked with the geo-database to facilitate the retrieval of individual objects and accompanying metadata (Figure 4).

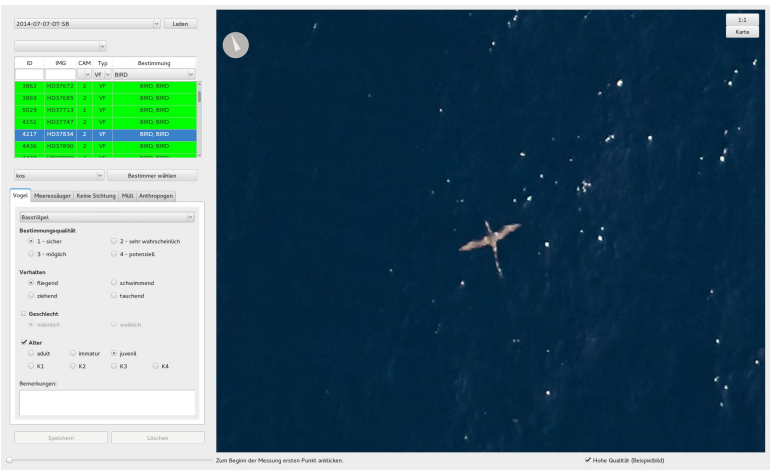

Figure 4. Screen shot of a software interface used for species identification (example image shows a juvenile gannet)

This ID tool also enables the measurement and storage of morphometric parameters, which is important supplementary information for identifying bird or mammal species (and which can be used to calculate the flight heights of birds, if relevant). If there are discrepancies in species identification between the two experts (as indicated automatically following a database query), a third expert can be consulted to review the case and issue a final decision. Typical features for the identification of bird species in aerial images have been described by Dittmann et al. (2014). All birds and marine mammals that are identified to genus or species level receive an attribute of accuracy. In the case of birds, individuals are further categorized (where possible) according to (1) sex, (2) age class (adult, immature, juvenile, K1-K4), and (3) behavioural traits (swimming, diving, flying, direction of flight). For marine mammals, additional information may be noted, e.g. the presence of calves.

After the ID process, the census information is transferred into standard data tables and used for the calculation of population densities at grid-cell or at survey-area level, and for geostatistical analyses related to habitat use (e.g. Skov et al., 2016) and potential displacement effects. Randomized samples of the photographic material containing classified georeferenced objects can be reciprocally quality controlled by external, independent reviewers. The geo-spatial positioning of individuals allows a precise measurement of their distance to anthropogenic structures (e.g. pipelines, offshore wind turbines) and relating species-specific distributions to functional ecological parameters (e.g. water depth), habitat features, and associated food resources.

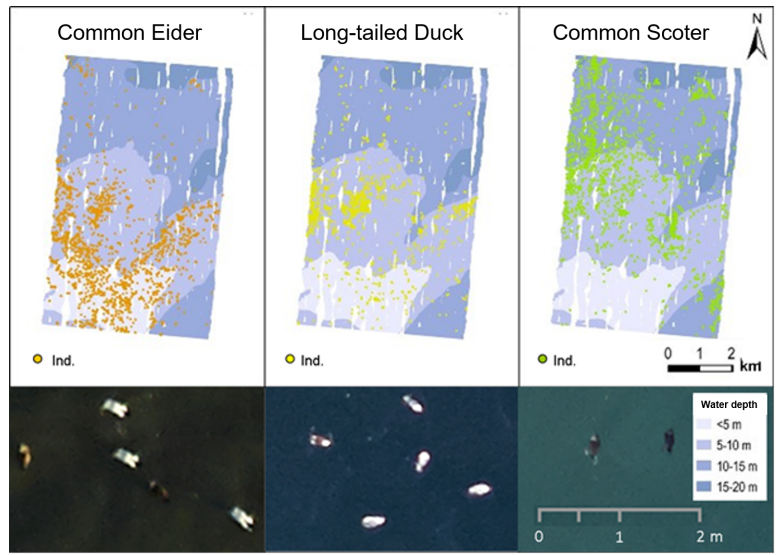

Figure 5. The distributions of three sea duck species based on gapless aerial photos taken on March 122014 in the German Baltic Sea (Bay of Wismar) in relation to different water depth classes. Maps are based on data by Steffen (2014).

The examples given in Figure 5 show the specific distributional patterns of three species of sea ducks in the Baltic Sea derived from aerial images covering almost $100 \%$ of the area (Steffen 2014). The morphological characteristics of these species as seen in aerial images (cf. Figure 5) are described by Dittmann et al. (2014) as follows:

Common Eider, Somateria mollissima. ô (nuptial plumage): white back, white scapulars and leg stain form typical "fish tail" shape; black tail, rump and flanks merge with dark background of the water surface; the black does not appear very clearly. $q$ : body is light brown on the light-facing side, the sides of the head contrasting in brighter beige.

Long-tailed Duck, Clangula hyemalis. $\hat{\alpha}$ (nuptial plumage): white plumage parts on head, nape and rump, and pale grey back plumage outshine darker plumage parts that merge partly with the background, giving the impression of a figure eight-type shape. : : white plumage parts on head and rump, which are separated by the dark back plumage, appear as two distinct spots. 
Common Scoter, Melanitta nigra. $\hat{\delta}$ (nuptial plumage): completely black; yellow patch on beak mostly invisible, feet as opposed to the Velvet Scoter Melanitta fuscata dark, but not always visible. $q$ : body completely dark brown, bright head sides contrasting strongly.

Databases containing this species-specific information coupled with individual biometric measurements will be useful when training automated object identification algorithms in order to accelerate the pre-screening process, which currently limits the overall workflow.

\section{CONCLUSIONS AND OUTLOOK}

The use of high-resolution aerial imagery to map marine wildlife shows several advantages over conventional observerbased methods. Site-specific frequencies of individuals can be determined without correcting for distance-related observation bias (Buckland et al., 2001), and the resulting population estimates remain verifiable at raw-data level. The method can also be applied to complement land-based waterbird counts, e.g. in protected areas, and to advance national or international monitoring schemes.

However, there are still a number of methodological challenges both in image acquisition an image analysis that need to be overcome in the future (Buckland et al., 2012; Taylor et al., 2014; Coppack et al. 2015). From a logistical and economic perspective, it is reasonable attempting to capture the full range of seabirds and marine mammal species with the same sensor technique, under the same sampling regime, and within the shortest possible time window. However, while white seabirds, gulls for example, are clearly visible against the dark background of the sea surface (in areas that are free of glare, glint, and spray), marine mammals often merge with their environment and may become visible only when they emerge from the sea surface and reflect the sunlight. These varying signal-to-noise relationships between biota require a high quantization of the imaging channels and filters, imposing equivalent requirements on the image processing software.

Up to now, seabirds and marine mammals have been usually targeted manually in aerial digital images. With the increasing regular use of aerial digital methods, image pre-processing (object recognition) should be automated in order to accelerate and standardise the entire workflow. Automated object recognition based on deep machine learning is subject to current research. Moreover, there is a need for further optimising sampling design and effort in view of the associated survey costs.

In principle, the following three parameters would need to be addressed: (1) the required minimum number of seasonal surveys to distinguish phenological and stochastic fluctuations from population changes (cf. Maclean et al. 2012); (2) the required size of a survey area to characterize habitat clines and their associated populations; (3) the minimum effective coverage of a survey area in conjunction with the optimum sampling design to obtain statistically robust results. These parameters are being continually discussed in several national and international research projects in order to define the minimum regulatory requirements for effective environmental impact assessments.

As mentioned above, glare is a critical factor that limits effective coverage, potentially producing many false positives. Glare effects show up in the RGB bands but not in the NIR. Current trials have therefore included a supplementary camera in the NIR band to obtain more information on glare detection and compensation. This could facilitate the automated separation of signals from background noise in order to detect objects of interest with higher precision and consistency.

Beside this improvement, a better image-to-image correlation as well as a better image rectification will be required. To achieve this with the setup of three cameras (two RGB and one NIR), further camera-calibrations using photogrammetric software are intended. We propose an accurate calibration of the cameras (calibration of focal length, PPS/PPA, radial distortion) and of the relative offset angles (relative boreside angles). A significant improvement could be achieved by using precise GNSS-INS to receive direct referencing values for the projection centres and the rotation angles Omega, Phi, and Kappa.

\section{REFERENCES}

Buckland, S. T., Anderson, D. R., Burnham, K. P., Laake, J. L., Borchers, D. L., Thomas, L., 2001. Introduction to Distance Sampling. Estimating abundance of biological populations. 4th Edition, Oxford University Press Inc., New York, USA.

Buckland, S. T., Burt, M. L., Rexstad, E. A., Mellor, M., Williams, A. E., \& Woodward, R., 2012. Aerial surveys of seabirds: the advent of digital methods. Journal of Applied Ecology, 49, pp. 960-967.

Coppack, T., Weidauer, A., Kemper, G., 2015. Erfassung von Seevogel- und Meeressäugerbeständen mittels georeferenzierter Digitalfotografie. AGIT, Journal für Angewandte Geoinformatik, 1, pp. 358-367.

Diederichs, A., Nehls, G. \& Petersen, I. K., 2002. Flugzeugzählungen zur großflächigen Erfassung von Seevögeln und marinen Säugern als Grundlage für Umweltverträglichkeitsstudien im Offshorebereich. Seevögel, 23 , pp. 28-46.

Dierschke, V., Exo, K. M., Mendel, B. \& Garthe, S., 2012. Gefährdung von Sterntaucher Gavia stellata und Prachttaucher G. arctica in Brut-, Zug- und Überwinterungsgebieten - eine Übersicht mit Schwerpunkt auf den deutschen Meeresgebieten. Vogelwelt, 133, pp. 163-194.

Dittmann, T., Fürst, R., Gebhardt-Jesse, U., Grenzdörffer, G., Kilian, M., Löffler, T., Mader, S., Schleicher, K., Schulz, A., Steffen, U., Weidauer, A. \& Coppack, T., 2014. Vogelbestimmung aus der Vogelperspektive. Vogelwarte, 52, pp. 335-336.

Garthe, S., Hüppop, O. \& Weichler, T., 2002. Anleitung zur Erfassung von Seevögeln auf See von Schiffen. Seevögel, 23, pp. 47-55.

Groom, G., Stjernholm, M., Nielsen, R. D., Fleetwood, A. \& Petersen, I. K., 2013. Remote sensing image data and automated analysis to describe marine bird distributions and abundances. Ecological Informatics, 14, pp. 2-8.

Grzimek, M. \& Grzimek, B., 1960. Flamingoes censused in east Africa by aerial photography. Journal of Wildlife Management, 24, pp. 215-217.

Kemper, G., 2012. New Airborne Sensors and Platforms for Solving Specific Tasks in Remote Sensing. ISPRS, International Archives of the Photogrammetry, Remote Sensing and Spatial Information Sciences, Vol. XXXIX-B5, pp. 351356. 
Kulemeyer, C., Schulz, A., Weidauer, A., Röhrbein, V., Schleicher, K., Foy, T., Grenzdörffer, G. \& Coppack, T., 2011. Georeferenzierte Digitalfotografie zur objektiven und reproduzierbaren Quantifizierung von Rastvögeln auf See. Vogelwarte, 49, pp. 105-110.

Maclean, I., Rehfisch, M. M., Skov, H., \& Thaxter, C. B., 2013. Evaluating the statistical power of detecting changes in the abundance of seabirds at sea. Ibis, 155, pp. 113-126.

Mendel, B. \& Garthe, S., 2010. Kumulative Auswirkungen von Offshore-Windkraftnutzung und Schiffsverkehr am Beispiel der Seetaucher in der Deutschen Bucht. Coastline Report, 15, pp. $31-44$

Müncheberg, R., Gosselck, F., Coppack, T. \& Weidauer, A., 2012. Klimawandel an der Ostsee: Interessenskonflikte zwischen Natur-und Küstenschutz bei der Gewinnung mariner Sande. In: Klimaanpassung als Herausforderung für die Regional- und Stadtplanung Erfahrungen und Erkenntnisse aus der deutschen Anpassungsforschung und -praxis. Mahammadzadeh, M., Chrischilles, E. (Eds.), Institut der Deutschen Wirtschaft, Cologne, Germany.

Skov, H., Heinänen, S., Thaxter, C. B., Williams, A. E., Lohier, S., \& Banks, A. N., 2016. Real-time species distribution models for conservation and management of natural resources in marine environments. Marine Ecology Progress Series, 542, pp. 221234.

Steffen, U., 2014. Entwicklung alternativer Sampling Designs bei der luftbildgestützten Seevogelzählung unter Berücksichtigung einer GIS-basierten Modellierung am Beispiel von Meeresenten. Master Thesis, Rostock University, Rostock, Germany.

Taylor, J. K. D., Kenney, R. D., Leroi, D. J. \& Kraus, S. D., 2014. Automated vertical photography for detecting pelagic species in multitaxon aerial surveys. Marine Technology Society Journal, 48, pp. 36-48.

Thaxter, B. C. \& Burton, N. H. K., 2009. High definition imagery for surveying seabirds and marine mammals: a review of recent trials and development of protocols. British Trust for Ornithology Report commissioned by COWRIE Ltd.

Thomsen, F., Laczny, M. \& Piper, W., 2004. Methodik zur Erfassung von Schweinswalen (Phocoena phocoena) und anderen marinen Säugern mittels Flugtransekt-Zählungen. Seevögel, 25, pp. 3-12. 